\title{
Diferenciación de cáncer gástrico temprano vs linitis plástica; ¿los síntomas hacen la diferencia?
}

\author{
Differentiation of early gastric cancer vs linitis \\ plastica: do symptoms make a difference?
}

\author{
Martín Alonso Gómez, Alejandro Concha, \\ William Alberto Otero • Bogotá, D.C. (Colombia)
}

\section{Resumen}

El cáncer gástrico tiene un patrón de presentación que va desde el estadio temprano a la linitis plástica, con pronósticos radicalmente diferentes, el primero con sobrevida a cinco años superior a $90 \%$, el segundo inferior a $5 \%$.

Objetivo: identificar si las características clínicas permiten diferenciar un estadío temprano de linitis plástica.

Metodología: se revisaron las bases de datos de la unidad de gastroenterología y endoscopia digestiva de un hospital de tercer nivel entre julio de 2005 y julio de 2010 , seleccionando los casos donde se encontró linitis plástica y cáncer gástrico temprano, todos confirmados por patología.

Resultados: se incluyeron 56 pacientes, 15 linitis y 41 cáncer temprano. Edad promedio de 61.3 años, (IC95\%54.5-68.2) para linitis y 63 años para cáncer temprano (39-85), 66.6\% fueron mujeres y $33.4 \%$ hombres en linitis plástica y $51.2 \%$ mujeres y $48.8 \%$ hombres en cáncer temprano. Compromiso del cuerpo en linitis $76.9 \%$, en cáncer temprano el cuerpo $29.3 \%$, antro $43.9 \%$. Pérdida de peso presentó un $73.3 \%$ en linitis y $26.8 \%$ en cáncer temprano. No hubo diferencias significativas en síntomas como: vómito, hematemesis, melenas o epigastralgia. El 26.8\% de los pacientes tenían antecedente de cáncer gástrico

Conclusion: los síntomas no son útiles como herramienta para diferenciar cáncer gástrico temprano de la linitis plástica, por lo cual se requieren otras estrategias para la detección del cáncer en estado temprano como podría ser la realización de EVDA en los pacientes con antecedentes familiares de este tumor. (Acta Med Colomb 2012; 37: 62-65).

Palabras clave: linitis plastica, cáncer gastrico, signos y síntomas digestivos.

\footnotetext{
Abstract

Gastric cancer has a pattern of presentation ranging from early stage to linitis plastica, with radically different prognosis; early cancer with 5 -year survival exceeding $90 \%$, and linitis plastica with survival of less than $5 \%$.

Objective: identify if the clinical features differentiate an early stage cancer from plastic linitis.

Methodology: we reviewed the databases of the unit of gastroenterology and endoscopy of a tertiary hospital between July 2005 to July 2010, selecting those cases where we found plastic linitis and early gastric cancer, all confirmed by pathology.

Results: we included 56 patients, 15 with linitis, and 41 with early cancer. Mean age was 61.3 years (95\% CI 54.5-68.2) for linitis and 63 years for early cancer (39-85). With plastic linitis, 66.6\% were female and $33.4 \%$ men, and with early cancer, $51.2 \%$ were women and $48.8 \%$ were men. Stomach body involvement in linitis was $76.9 \%$; in early cancer, body involvement was $29.3 \%$; antrum involvement was $43.9 \% .73 .3 \%$ of patients with linitis had weight loss and $26.8 \%$ had it with early cancer. There were no significant differences in symptoms such as vomiting, hematemesis, melena or epigastralgia. $26.8 \%$ of patients had a history of gastric cancer.
}

Dr. Martín Alonso Gómez Zuleta: Médico internista, Gastroenterólogo, Profesor de Gastroenterología, Universidad Nacional de Colombia, Gastroenterólogo, Unidad de Gastroenterología y Endoscopia Digestiva del Hospital El Tunal ESE, Bogotá; Dr. Alejandro Concha Mejía: Médico Internista, Epidemiólogo, Fellow de Gastroenterología, Universidad Nacional de Colombia; Dr. William Alberto Otero Regino: Médico Internista, Gastroenterólogo, Profesor de Gastroenterología y Jefe de Postgrado de Gastroenterología, Universidad Nacional de Colombía. Bogotá, D.C. (Colombia) Correspondencia: Dr. Alejandro Concha E-mail: alcome@gmail.com Recibido: 16/VII/2011 Aceptado: 01/VI/2012 
Conclusion: the symptoms are not useful as a tool to differentiate early gastric cancer from linitis plastica, so other strategies are required for detection of early stage cancer, such as the performance of endoscopy in patients with a family history of this tumor. (Acta Med Colomb 2012; 37: 62-65).

Keywords: linitis plastica, gastric cancer, signs y symptoms digestive.

El cáncer gástrico cuya carga de morbimortalidad es ampliamente conocida en nuestro país, tiene un patrón de presentación muy variable que va desde el estadio temprano a la linitis plástica. El pronóstico es radicalmente diferente dado que el primero tiene una sobrevida a cinco años superior a $90 \%$ mientras que el segundo es inferior a $5 \%$ (1), por lo cual es de gran importancia la detección de estos tumores en su estado temprano. Infortunadamente en nuestro medio, la detección de cáncer gástrico en estadio temprano solo se logra en menos de $10 \%$ de los casos a diferencia de Japón donde puede ser superior a 50\% (1). De otro lado la linitis plástica es el peor espectro de la enfermedad y se presenta en 3-19\% de los casos (2). Aunque estos dos son los polos opuestos de la enfermedad, sería muy importante conocer su comportamiento, y así poder mejorar el pronóstico de esta entidad. Pero para lograr este objetivo es necesario conocer el comportamiento clínico de estos tumores para identificar las características que pudieran orientar para un diagnóstico temprano y cuáles orientarían al diagnóstico de la linitis plástica, con el objetivo de implementarlas en un programa de detección temprana.

En la linitis plástica el comportamiento clínico y biológico implica que aun con resecciones curativas la sobrevida sea corta (3). La sobrevida acumulada va del 2 al 10\% (4), siendo la linitis por sí sólo un factor pronóstico independiente en la sobrevida en cáncer gástrico (5).

El cáncer gástrico temprano corresponde al otro polo de la enfermedad, con una sobrevida que supera el $90 \%$ y en países con programas de tamización ("screening") representa el $65 \%$ de todos los cánceres gástricos (6).

Los factores de riesgo conocidos para cáncer gástrico son los mismos para el cáncer temprano y la linitis plástica, sin embargo otros factores como el factor de crecimiento transformante factor de crecimiento transformante ( $\beta 1$ - TGF- $\beta 1$ ) (7); factor de crecimiento endotelial vascular VEGF (8) y mutaciones en el gen de la E-cadherina (9), se asocian a la linitis plástica.

Las presentaciones clínicas muestran los extremos de la enfermedad, mientras el cáncer temprano en la práctica diaria, se encuentra de manera incidental, en pacientes con dispepsia no investigada o en casos de endoscopia por otros síntomas, en cambio en la linitis plástica los síntomas son severos y notorios, reflejando el carácter avanzado de la enfermedad (10).

El tratamiento del cáncer temprano es endoscópico, bien sea con resección endoscópica o mucosectomía o con disección endoscópica de la submucosa, con lo cual se logra una tasa de sobrevida a cinco años cercana al $100 \%$ como recientemente lo demostramos en nuestro medio y que es similar a lo encontrado en otros países (11). En cuanto a la linitis plástica la mayoría de los casos en nuestro medio termina en medidas paliativas y sólo unos pocos casos en cirugía y quimioterapia. Esta última ha mostrado resultados prometedores, pero no modifica sustancialmente la sobrevida (12).

\section{Material y métodos}

Se revisaron las bases de datos de la unidad de gastroenterología y endoscopia digestiva del Hospital El Tunal de Bogotá, una institución de salud de tercer nivel, entre julio de 2005 y julio de 2010, seleccionando los casos en los que como diagnóstico endoscópico se encontró linitis plástica o Borrmann IV y cáncer gástrico temprano, todos confirmados por patología. Se realizó revisión activa de historias clínicas y patologías, se tomaron los datos en un cuestionario estructurado, los datos clínicos y fechas de muerte se completaron por medio de llamadas telefónicas confidenciales con formato de llamada prediseñado. A todos los pacientes se les realizó TAC de abdomen para estadificación. Los datos se analizaron SPSS 15.0. Este estudio cumple con aprobación del comité de ética y de investigaciones de la institución y con la Resolución del Ministerio de Salud 8430 de 1993, siendo investigación sin riesgo.

\section{Resultados}

Durante el periodo de estudio de cinco años se lograron incluir 56 pacientes, 15 con linitis plástica y 41 con cáncer gástrico temprano. La edad promedio fue de 61.3 años, con un rango entre 40 y 95 años (IC 95\% 54.5 68.2) para linitis plástica, y de 63 años para cáncer gástrico temprano con un rango de 39 a 85 años, la edad promedio en hombres fue de 61.9 años para linitis plástica y 63 años para cáncer temprano y en mujeres de 61 años en linitis plástica y 62 años en cáncer temprano. El sexo se distribuyó con un $66.6 \%$ mujeres y $33.4 \%$ hombres en linitis plástica y $51.2 \%$ mujeres y $48.8 \%$ hombres en cáncer gástrico temprano. La región de procedencia con más casos fue Bogotá con $66.6 \%$, seguida en su orden por Boyacá y Pasto.

En relación con el sitio de localización de la lesión gástrica en los pacientes con linitis plástica $76.9 \%$ correspondió al cuerpo, $11.5 \%$ al antro y a la presencia extendida de cuerpo y antro. En los de cáncer temprano en el fondo se localizaron $17.1 \%$, en el cuerpo 29.3 , en el antro $43.9 \%$, y en $9.7 \%$ la localización fue cardial.

El tipo de cáncer temprano se distribuyó así: tipo I $22.2 \%$, tipo IIa $48.1 \%$, tipo IIb $3.7 \%$, tipo IIc $22.2 \%$ y el 
tipo III $3.7 \%$, en hombres tipo I $33.3 \%$, tipo IIa $41.7 \%$, tipo IIb $8.3 \%$, tipo IIc $16.7 \%$ sin casos de tipo III, en las mujeres tipo I $13.3 \%$, tipo IIa $53.3 \%$, tipo IIb no presentó casos, tipo IIc $26.7 \%$ y el tipo III un $6.7 \%$.

Los síntomas presentados y susceptibles de análisis fueron pérdida de peso, epigastralgia de cualquier tipo, náusea o vómito y hematemesis o melenas, encontrando que la pérdida de peso se presentó en $73.3 \%$ de los pacientes con linitis plástica y sólo $26.8 \%$ en los de cáncer temprano, con un $\mathrm{LR}+$ de 2.7 ( $\mathrm{p}=0.037)$ como factor predictivo de tener un cáncer avanzado y no uno temprano. La epigastralgia de cualquier tipo en linitis o cáncer temprano se presentó en $80 \%$ y $80.4 \%$ respectivamente, $\mathrm{LR}+1$, ( $\mathrm{p}=\mathrm{NS}$ ), el grupo de síntomas comprendidos en náusea o vómito se presentó en $60 \%$ en linitis plástica y $80.4 \%$ en cáncer temprano, $\mathrm{LR}+1.7$ ( $\mathrm{p}=\mathrm{NS}$ ); hematemesis o melenas se presentaron en $46.6 \%$ en linitis plástica y $26.8 \%$ para cáncer temprano, LR+ 1.7 ( $\mathrm{p}=\mathrm{NS}$ ) (Tabla 1).

En cuanto a la estratificación encontramos que todos los pacientes con cáncer temprano se hallaban en estadio I, sin evidencia de metástasis en el TAC de abdomen o compromiso ganglionar, la ecoendoscopia se pudo realizar a 15 de los 41 pacientes con CA temprano, estableciendo en todos que el tumor estaba limitado a la submucosa. En todos los pacientes con linitis plástica la TAC abdominal encontró que estaban en estadio IV dada la presencia de ascitis o metástasis hepáticas.

En el grupo de linitis plástica sólo se encontraron dos casos con antecedentes familiares de cáncer gástrico y en el de cáncer gástrico temprano se presentaron 11 casos correspondiendo a $26.8 \%$. No fue posible establecer datos se sobrevida media en los casos estudiados.

\section{Discusión}

Dada la alta morbimortalidad del cáncer gástrico en nuestro país, este debería ser tratado como un verdadero problema de salud pública y como tal tener la ingerencia permanente del Estado (13). En el cáncer gástrico hay dos polos de la presentación nosológica que son tan disímiles en sobrevida, cuadro clínico y tratamiento, compartiendo sólo la mayoría de factores de riesgo de la patología, que hacen necesario establecer cuáles características de las mencionadas nos orienten y nos alerten hacia la presencia en sí de uno de ellos (temprano o linitis).

Los síntomas como parte del diagnóstico de cáncer gástrico sólo aparecen cuando la enfermedad es avanzada y esto es evidente por el análisis de las razones de probabilidades aplicadas a cada grupo de síntomas presentes en los pacientes estudiados, demostrando que solamente la pérdida significativa de peso tiene un LR+ con significancia estadística. Aunque en el análisis se han encontrado que otros signos clínicos como vómito y la presencia de melenas o hematemesis definidos clásicamente como de alarma, que según lo expuesto en diferentes publicaciones orienta a enfermedad neoplásica, tuvieron razones de probabilidad
Tabla 1. Síntomas según tipo de cáncer.

\begin{tabular}{|l|c|c|c|c|c|}
\hline Sintoma & Linitis & Temprano & LR+ & LR- & $\mathbf{p}$ \\
\hline Pérdida de peso & $11 / 15$ & $11 / 41$ & 2.7 & 0.36 & 0.037 \\
\hline Epigastralgia & $12 / 15$ & $33 / 41$ & 1 & 1 & NS \\
\hline Náusea o vómito & $9 / 15$ & $14 / 41$ & 1.7 & 0.6 & NS \\
\hline Hematemesis o melenas & $7 / 15$ & $11 / 41$ & 1.7 & 0.7 & \\
\hline NS: no significativa.
\end{tabular}

positiva que sugieren la presencia de cáncer avanzado, sin tener significancia estadística, lo cual nos sugiere que los síntomas no son predictores clínicos para diferenciar los polos de la enfermedad, por lo cual no serían útiles para poder detectar los casos tempranos, lo cual nos obliga a buscar otro tipo de armas para luchar contra la enfermedad al intentar detectarla en estado temprano (marcadores bioquímicos, EVDA en asintomáticos, etc.)

La edad no presentó diferencias entre los dos tipos de cáncer; sin embargo, hubo una leve diferencia con mayor edad en los casos de cáncer gástrico temprano (14). Contrario a lo encontrado por An et al. en 2008, la edad de diagnóstico de la linitis plástica es menor aproximadamente por 10 años a lo encontrado en nuestro estudio, sin embargo sabemos las diferencias en cuanto a acceso a servicio de salud y programas relacionados con cáncer gástrico en países como Japón que podrían explicar las diferencias descritas, de la misma manera el hecho de que en nuestro estudio las mujeres sean el grupo predominante casi duplicando a los hombres en linitis plástica, contrasta con los datos del mismo estudio de referencia planteando que las mujeres son un grupo de riesgo para sufrir el espectro más avanzado de la enfermedad; sin embargo, somos conscientes de que este tipo de aseveraciones requieren de otros diseños epidemiológicos para demostrar la causalidad.

La ubicación gástrica de las linitis plástica fue predominantemente corporal, lo que es claramente similar a lo encontrado en algunas series de casos como la de An et al, que mostró compromiso de tercio superior en $14.2 \%$, tercio medio en $31.1 \%$ e inferior en $54.3 \%$, marcando la diferencia la presencia de una carga muy alta asignada a la localización en cuerpo (76.9\%) que supera en sí los hallazgos de los estudios mencionados.

En este sentido la búsqueda de signos de alarma es mandatoria en los pacientes con sintomatología gastrointestinal; sin embargo, no es una actividad clínica que disminuya la mortalidad, de los pacientes con neoplasia gástrica ya que cuando estos están presentes nos indicaría que es una enfermedad avanzada, que como se sabe tiene mínimas posibilidades de intervención curativa.

Extrapolando esta información al terreno de la prevención consideramos que los resultados de este estudio, el cual es el primero que compara los dos polos de la enfermedad, demuestran que no son los marcadores clínicos las herramientas más adecuadas para hacer una tamización 
en el cáncer de cáncer gástrico, no permitiría intervención temprana la cual es una de las metas y razones de ser de la tamización, sino que da fuerza, a que sea masificando la endoscopia digestiva superior la única herramienta disponible para realmente impactar la carga de morbimortalidad del cáncer gástrico en nuestro medio. De la misma manera el encontrar que uno de cada cuatro pacientes con cáncer temprano tenía antecedentes familiares de cáncer gástrico nos indicaría que posiblemente éste sería uno de los grupos de pacientes en los que deberían realizarse exámenes endoscópicos de forma más temprana. Otra estrategia que puede utilizarse para detectar tempranamente estos tumores es la realización de la endoscopia a pacientes mayores de 30 años con o sin síntomas como lo demostramos en un trabajo recientemente (15).

\section{Conclusiones}

Los síntomas no permiten discriminar de manera satisfactoria un cáncer gástrico avanzado de un cáncer gástrico temprano curable. Por lo tanto, consideramos que lo mejor sería realizar endoscopia digestiva en pacientes adultos con dispepsia.

\section{Conflictos de interés}

Los autores niegan conflictos de interés.

\section{Referencias}

1. Tsukuma H, Oshima A, Narahara H, Morii T. Natural history of early gastric cancer: a non-concurrent, long term, follow up study. Gut 2000; 47(5): 618-21.
2. Kitamura K, Beppu R, Anai H, Ikejiri K, Yakabe S, Sugimachi K, et al. Clinicopathologic study of patients with Borrmann type IV gastric carcinoma. $J$ Surg Oncol 1995; 58(2): 112-7.

3. Nakamura R, Saikawa Y, Wada N, Yoshida M, Kubota T, Kumai K, et al. Retrospective analysis of prognosis for scirrhous-type gastric cancer: one institution's experience. Int J Clin Oncol 2007; 12(4): 291-4.

4. Arveux P, Faivre J, Boutron MC, Piard F, Dusserre-Guion L, Monnet E, et al Prognosis of gastric carcinoma after curative surgery. A population-based study using multivariate crude and relative survival analysis. Dig Dis Sci 1992; 37(5): 757-63.

5. An JY, Kang TH, Choi MG, Noh JH, Sohn TS, Kim S. Borrmann type IV: an independent prognostic factor for survival in gastric cancer. J Gastrointest Surg 2008; 12(8): 1364-9.

6. Leung W, Enders K, Sung J, Yamada T. Tumors of the stomach Textbook of gastroenterology 5 ed. Oxford, Blackwell publishing; 2009. p. 1026-1053.

7. Kinugasa S, Abe S, Tachibana M, Hishikawa Y, Yoshimura H, Monden N, et al. Overexpression of transforming growth factor-beta1 in scirrhous carcinoma of the stomach correlates with decreased survival. Oncology 1999; 55(6): 582-7.

8. Baba M, Konno H, Maruo Y, Tanaka T, Kanai T, Matsumoto K, et al. Relationship of p53 and vascular endothelial growth factor expression of clinicopathological factors in human scirrhous gastric cancer. Eur Surg Res 1998; 30(2): 130-7.

9. Pedrazzani C, Corso G, Marrelli D, Roviello F. E-cadherin and hereditary diffuse gastric cancer. Surgery 2007; 142(5): 645-57.

10. Clark CJ, Thirlby RC, Picozzi V, Jr., Schembre DB, Cummings FP, Lin E Current problems in surgery: gastric cancer. Curr Probl Surg 2006; 43(8-9): $566-$ 670 .

11. Gomez M, Otero W, Arbelaez V. Five year monitoring of endoscopic treatment for early gastric cancer in Colombia. Rev Col Gastroenterol 2009; 24(4): 347-352.

12. Kinoshita T, Sasako M, Sano T, Katai H, Furukawa H, Tsuburaya A, et al. Phase II trial of S-1 for neoadjuvant chemotherapy against scirrhous gastric cancer (JCOG 0002). Gastric Cancer 2009; 12(1): 37-42.

13. Otero W. Cáncer gástrico en Colombia: un diagnóstico tardìo que exige compromiso del Estado (Editorial). Rev Col Gastroenterol 2008; 23: 302-6.

14. Everett SM, Axon AT. Early gastric cancer in Europe. Gut 1997; 41(2): 142-50.

15. Pineda LF, Otero W, Gómez M, Arbeláez V, Otero E. Enfermedad estructural y valor predictivo de la historia clínica en pacientes con dispepsia no investigada. Revax Col Gastroenterol 2004; 19(1): 13-25 